\title{
Ueber eine einfache, therapeutisch kräftige, Methode der Anwendung von Unguentum Hydragyri.
}

\author{
Von \\ Professor Edvard Welander in Stockholm.
}

$\mathrm{Zu}$ dem 2. internationalen Congress in Wien im Jahre 1892 hatte ich zwei Vorträge angemeldet: „Untersuchungen über die Absorption und Elimination des Quecksilbers bei der unter verschiedenen Verbältnissen ausgeführten Einreibungscur" und "Ueber die Behandlung von Syphilis mittels Ueberstreichens - nicht Einreibens - von Quecksilber".

Leider war ich dann verhindert den Congress zu besuchen. Meine beiden Vorträge wurden nachher in das Archiv für Dermatologie und Syphilis 1893 aufgenommen. Ich suchte in ihnen eine Menge Gründe dafür darzulegen, dass $\mathrm{Hg}$ bei gewöhnlicher Zimmertemperatur verdunstet und dann in grosser Menge vom Menschen absorbirt werden kann; ich suchte den Beweis zu liefern, dass bei der Einreibungscur die grösste Menge $\mathrm{Hg}$ in dieser Weise uud nicht durch die Einreibung in die Haut in den Körper gelangt; ich schlug vor, bei der Anwendung von $\mathrm{Hg}$ die Salbe deshalb nicht in die Haut einzureiben, sondern sie nur über dieselbe zu streichen, und ich berichtete, dass ich in einer bedeutenden Anzahl von Fällen durch dieses Verfahren eine kräftige $\mathrm{Hg}$-Absorption und sehr gute therapeutische Ergebnisse erhalten hatte. (Meine Erfahrung ist später von Lesser, Lang u. A., welche meine Methode angewandt haben, bekräftigt worden.)

Es lag ja nahe, sich zu fragen, ob es unter diesen Verhältnissen unbedingt nothwendig sei, die Salbe über die Haut zu streichen, was ja stets etwas unsauber und für den Patienten unangenehm ist. Ich zeigte, dass dieses nicht der 
Fall ist, denn wenn ich Wachstaffet mit Ung.-Hg bestrich und ihn dann in solcher Weise applicirte, dass die Salbe nicht mit der Haut in Berührung kommen konnte, gleichwobl eine sebr starke Absorption von $\mathrm{Hg}$ stattfand. Es ist ja selbstrerständlich, dass ich unter solchen Umständen nach einer praktischen Methode suchte, die Salbe so zu appliciren, dass sie mit der Haut nicht in directe Berührung zu kommen braucht. Ich habe in dieser Richtung zahlreiche Versuche ausgeführt, aber erst vor einigen Monaten habe ich eine besonders einfache Methode gefunden, die Salbe in dieser Weise anzuwenden.

Um eine kräftige Absorption zu erhalten, musste ich, wie meine Versuche mich gelehrt hatten, die Salbe, damit ich eine möglichst grosse Abdünstungsfläche bekomme, über eine grosse Fläche ausbreiten und sie dann bei einer nicht allzu niedrigen Temperatur erhalten. Ich wandte ja deshalb die Ueberstreichung auf den Körpertheilen an, die Sigmund vorgeschrieben hat, und liess dann den Patienten 10-14 Stunden in Bettwärme liegen.

Dieses Verfahren kann nun in einfacher Weise dadurch ersetzt werden, dass man einen kleinen Sack, z. B. ein kleines Kopfkissen, nimmt, in diesem Sack sorgfältig die Salbe mit einem Spatel ausbreitet und ihn dann auf die Brust oder den Rücken legt. Diesen kleinen Sack habe ich in der Länge von ungefähr 50 und der Breite von ungefähr 40 Centimeter angewandt. Đadurch, dass man an dem oberen und dem unteren Theile dieses Sackes je zwei Bänder anbringt, die am oberen Theile angebrachten über die Achseln zieht, auf dem Rücken kreuzt und dann, gleich unterhalb der Achseln, auf der Brust und die am unteren Theile befestigten auf dem Kreuze zusammenbindet, wird er ausgebreitet erhalten und sein Zusammenfalten verhindert. Anfangs wandte ich täglich $8 \mathrm{Gr}$. Ung.-Hg $(1: 3)$ an, doch sind 6 Gr. rollständig hinreichend; ebenso bestrich ich anfangs den Sack täglich mit neuer Salbe, was ebenfalls nicht nothwendig ist, und die Patienten hatten ihn den ganzen Tag über auf dem Körper, während sie ihn nur in der Bettwärme (10-14 Stunden) zu haben brauchen. Wenn der Sack einige Tage angewandt worden ist, dringt die Salbe durch und macht ihn schmutzig; will man, so kann wan ihn 
Ueb. e. einf., therap. kräft. Methode d. Anw. v. Unguentum Hydr. $2 \overline{5} 9$

nun gegen einen reinen umtauschen, doch kann dieses auch unterbleiben, da ihn der Patient ohne die geringste Ungelegenheit 30 Tage lang haben kann.

Fragen wir nun, ob in dieser Weise wirklich $\mathrm{Hg}$ absorbirt wird, was sich mit vollem Grunde vermuthen liess, so will ich als Antwort nur eine einzige Serie meiner in dieser Richtung ausgeführten Untersuchungen darlegen.

J., Frau, 18 Jahre. Bekam am 1./5. 1897 den Sack zu tragen ( $8 \mathrm{Gr}$. tägl.).

Den 1./5. in 345 Gr. Harn 1.020 keine Hg-Kügelchen.

"6./5. "355" " 1.022 eine Menge $\mathrm{Hg}-$ Kügelchen, einige nicht kleine.

$" 10 . / 5 . \quad 355 \quad " \quad$ " 1.012 eine bedeutende Menge meist kleiner $\mathrm{Hg}$-Kügelchen.

"15./5. "375 " "1.021 eine bedeutende Menge meist kleiner Hg-Kügelchen.

"20./5. $\quad 370, \quad \# 1.020$ zwei sehrgrosse und eine grosse Menge kleiner $\mathrm{Hg}$-Kügelchen. $" 28.5 . \quad 365 \Rightarrow \quad " 1.015$ eine grosse Menge grosser und zieml. grosser $\mathrm{Hg}$-Kügelchen. "30./5. "365 " " 1.020 eine bedeutende Menge ziemlich grosser $\mathrm{Hg}$-Kügelchen. "4./6. " $360 \quad$, 1.018 eine kolossale Menge grösserer und kleinerer $\mathrm{Hg}$-Kügelchen.

Wir sehen, wie kräftig und schnell hier die Absorption von $\mathrm{Hg}$ gewesen ist. Ganz gleich hat sich das Verhältniss in sämmtlichen von mir untersuchten 20 Fällen gezeigt. Einige Male habe ich die Hg-Kügelchen mit dem blossen Auge sehen können (Spiegel), so z. B. in dem oben angeführten Fall am 4./6. Nur bei der Einspritzung ron Calomel (in der von Smirn off zuerst bescbriebenen Weise) habe ich eine so grosse Menge $\mathrm{Hg}$ im Harn gefunden.

Dass bei einer so kräftigen Absorption von $\mathrm{Hg}$ die therapeutischen Ergebnisse gut gewesen sind, versteht sich von selbst; die Symptome sind schnell verschwunden. Dass ich die Fälle nicht ausgewäblt habe, geht aus den Symptomen bei den ersten der ron mir in dieser Weise behandelten 20 Patienten hervor: 3 hatten Sclerose und Adenitis (ohne allgemeine 
Symptome), 5 Roseola, Papulae mucosae u. s. w., 6 papulöses Syphilid (3 davon Psoriasis plant. und palmar.), I papulopustulöses Syphilid, 1 Papulo-Tuberkeln und 2 ulcerirende Gummata. In allen diesen Fällen verschwanden die Symptome schnell, und ich glaube nicht, dass ich mit irgend einer anderen Behandlung bessere und schnellere Ergebnisse erhalten haben würde.

Fragen wir nach den Ungelegenheiten, welche diese Behandlung mit sich führt, so hat sich in 4 Fällen eine gelinde Stomatitis und in nicht weniger als 6 Fällen eine gelinde Albuminurie (nur mit Trichloressigsäure nachweisbar) und Cylindrurie gezeigt; dieses kann ja bei einer so kräftigen $\mathrm{Ab}$ sorption (Elimination) von $\mathrm{Hg}$ nicht Wunder nehmen - eine solche Hg-Albuminurie hat ja keine Bedeutung. - In zwei Fällen ist nach der Anwendung des Sackes eine minimale Reizung der Haut aufgetreten, welche Reizung aber, sobald der Patient den Sack, anstatt auf die Brust, auf den Rücken band, innerhalb 24 Stunden verschwand. Die Ungelegenhei'sen sind also bei dieser Behandlungsweise sehr unbedeutend gewesen.

Ist nun diese Methode für die Behandlung eines einzelnen Ausbruches der Syphilis geeignet, so ist sie es in einem umso höheren Grade für eine intermittente, präventive Behandlung. Ich kann ja diese Methode ohne Reizung der Haut, der Hinterbacken, des Darmcanales u. s. w. - ja ich kann sagen ohne Ungelegenheit für den Patienten - anwenden wann und wie ich will.

Einen Theil von Details und der von mir gemachten Beobachtungen hoffe ich später mittheilen zu können.

Der Anlass, dass ich schon jetzt diese Untersuchungen darlege, ist der, dass Merget, wie ich aus Jadassohn's Besprechung seiner Arbeit im Archiv für Dermatologie und Syphilis ersehen habe, schon ror mehreren Jahren eine im Princip ähnliche Methode wie die nun von mir versuchte angewandt hat. Ich habe erst jetzt (Ende Juni) Gelegenheit gehabt, Merget's Buch durchzulesen. Auch wenn sich darin Vieles findet, was man nicht unterschreiben kann, so muss man doch Merget's Arbeit bewundern und darüber erstaunen, 
Ueb. e. einf., therap. kräft. Methode d. Anw. v. Unguentum Hydr. 261

dass seine Methode, ungeachtet sie von ihm schon vor mehr als einem Decennium dargelegt worden ist, nicht einmal in Frankreich Anklang gefunden hat.

In einem künftigen Aufsatz werde ich die Gründe darlegen, weshalb ich die von mir angewandte Methode gleichwohl für einfacher als Merget's (flanelles mercurielles) ansehe, welche mir, wie ich schon oben angedeutet, erst jetzt bekannt geworden ist. Hätte ich Merget's Methode schon früher gekannt, so würde sie bei der Ansicht, die ich mir durch eigene Untersuchungen über die Absorption des Quecksilbers gebildet habe, schon längst von mir versucht worden sein. 